\title{
Determinação de Coeficientes de Actividade de soluções electrolíticas pelas relações de Debye-Hückel
}

O modelo de Debye-Hückel para soluçð̃es electrolíticas pretende justificar o afastamento a um comportamento ideal; baseia-se num tratamento estatístico de sistemas onde se consideram determinado tipo de interacções, advenientes das forças iónicas. Apesar das suas limitaçð̃es intrínsecas, é de aplicação generalizada, sendo matéria corrente dos programas curriculares. Vencidas as dificuldades de carácter matemático, inerentes à formulação teórica, o estudante é, usualmente, conduzido a relações que possibilitam o cálculo de coeficientes de actividade iónicas médios*.

$O$ presente trabalho tem como objectivo realçar alguns aspectos importantes, geralmente omissos na exposição do assunto, sem os quais os valores obtidos são passíveis de interpretação indevida.

Considerando, por simplicidade, uma solução binária, os potenciais químicos do solvente, $\mu_{\mathrm{S}}$, e do soluto, $\mu_{\mathrm{S}}$, são expressos em função das respectivas actividades, a e $a_{s}$,

$\mu_{\mathrm{S}}=\mu \mathrm{\rho}+\mathrm{RT} \ln \mathrm{a}_{\mathrm{S}}=\mu \mu_{\mathrm{S}}+\mathrm{RT} \ln \mathrm{f}_{\mathrm{S}} \mathrm{x}_{\mathrm{S}}$

$\mu_{\mathrm{s}}=\mu_{\mathrm{s}}^{\ominus}+\mathrm{RT} \ln \mathrm{a}_{\mathrm{s}}=\mu_{\mathrm{s}}^{\ominus}+\mathrm{RT} \ln \mathrm{f}_{\mathrm{s}} \mathrm{x}_{\mathrm{s}}$

onde $\mu^{\Theta}$ representa o potencial químico do estado padrão, $\mathrm{x}$ a concentração em fracção molar e f o factor correctivo, coeficiente de actividade. Este traduz o afastamento da solução real do caso de idealidade

$\mu-\mu^{\circ}=\mathrm{RT} \ln \mathrm{f}$,

onde $\mu^{\circ}$ é o potencial químico da situação ideal.

Recorrendo às escalas práticas de concentração, molal, m, e molar, c, (por razð̄es bem conhecidas deve privilegiar-se a primeira), a relação (2) é, respectivamente, expressa por

$\mu_{\mathrm{s}}=\mu_{\mathrm{m}, \mathrm{s}}^{\ominus}+\mathrm{RT} \ln \gamma_{\mathrm{s}} \mathrm{m}_{\mathrm{s}}$

ou

$\mu_{\mathrm{s}}=\mu_{\mathrm{c}, \mathrm{s}}^{\Theta}+\mathrm{RT} \ln \mathrm{y}_{\mathrm{s}} \mathrm{C}_{\mathrm{s}}$

onde $\mu \stackrel{\ominus}{\mathrm{m}, \mathrm{s}}$ e $\mu \stackrel{\theta, s}{\theta_{c}}$ são os potenciais dos estados padrão, $\gamma_{\mathrm{s}}$ e $y_{\mathrm{s}}$ os coeficientes de actividade do soluto nas respectivas escalas.

No caso dum electrólito $\mathrm{M}_{\vartheta} \mathbf{X}_{\vartheta}{ }^{-}$em solução, o potencial químico do soluto, $\mu_{\mathrm{s}}$, advém da aditividade dos potenciais relativos às espécies iónicas i, j, $\mu_{\mathrm{s}}=\mu_{\mathrm{i}^{+}}+\mu_{\mathrm{j}^{-}}$

conduzindo à relação

$\mu_{\mathrm{s}}=\mu{ }_{\mathrm{s}}^{\theta}+\vartheta \mathrm{RT} \ln \vartheta_{ \pm} \mathrm{f}_{ \pm} \mathrm{x}$

ou, nas escalas práticas, removendo por simplicidade o indice $s$,

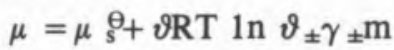

$\mu=\mu \stackrel{\theta}{\mathrm{c}}+\vartheta \mathrm{RT} \ln \vartheta_{ \pm} \gamma_{ \pm} \mathrm{c}$

obtidas através da introdução das grandezas médias

$\vartheta \pm=\left(\vartheta_{+} \vartheta_{-}\right)^{1 / \vartheta}$

$\mathrm{f} \pm=\left(\mathrm{f}_{+}^{\vartheta+} \mathrm{f}^{\vartheta-}\right)^{1 / \vartheta}$

e de

$\vartheta=\vartheta_{+}+\vartheta_{-}$

Os coeficientes de actividade podem ser determinados por via experimental, recorrendo a qualquer técnica que, em princípio, meça directa ou indirectamente o potencial químico, seja do soluto, seja do solvente, face ao inter-relacionamento existente pela equação de Gibbs-Duhem

$\mathrm{X}_{\mathrm{S}} \mathrm{d} \mu_{\mathrm{S}}+\mathrm{X}_{\mathrm{s}} \mathrm{d} \mu_{\mathrm{S}}=0$

Naturalmente, em casos concretos, a opção experimental recai sobre o método que propicie a maior simplicidade e rigor, condicionada pela solubilidade do electrólito, gama de concentração, natureza do solvente, temperatura e pressão do sistema.

Pela sua importância, como forma independente de avaliar a actividade do soluto e como complemento da informação obtida através de medidas isopiésticas do solvente, dentro das determinaçðes directas destaca-se a Potenciometria - implicando a existência de eléctrodo reversível adequado.

O sistema mais rigoroso é

$\mathrm{Pt}, \mathrm{H}_{2}\left|\mathrm{H}_{(\mathrm{m})}^{+} \mathrm{Cl}^{-}\right| \mathrm{Ag} \mathrm{Cl}, \mathrm{Ag}$

obtendo-se o coeficiente de actividade através da medição da f.e.m., E, da célula (14),

$\mathrm{E}=\mathrm{E}^{\circ}-\frac{\mathrm{RT}}{\mathrm{F}} \ln \mathrm{m}_{\mathrm{H}^{*}} \mathrm{~m}_{\mathrm{Cl}^{-}} \gamma_{ \pm}^{2}$

A reprodutividade dos resultados é da ordem de $\pm 0,02$ $\mathrm{mV}$, a que corresponde um erro de 2 na 4 . $^{\mathrm{a}}$ casa decimal de $\log \gamma_{ \pm}$, i.é., $\pm 0,05 \%$ em $\gamma_{ \pm}$. Este rigor é, no entanto, condicionado pelo da preparação das soluções

\footnotetext{
* A teoria permite o cálculo de coeficientes individuais. No entanto, a compatibilização com a realidade experimental justifica o critério aceite
} 
e do conhecimento das suas concentraçðes, temperatura e ruído do equipamento. Outros sistemas fornecem valores de $\mathrm{E}$ menos rigorosos. Mesmo com uma aproximação de $\pm 1 \mathrm{mV}$ obtém-se o coeficiente de actividade com um erro de $\pm 2,5 \%$, muitas vezes suficiente para testar aspectos teóricos e, com certeza, melhor aproximação que considerar $\gamma_{ \pm}=1$, isto é, um comportamento ideal.

Nos métodos indirectos, a técnica isopiéstica, baseada em diferenças da pressão de vapor no solvente (água), é a mais vulgarizada. O coeficiente de actividade do solvente é calculado a partir do coeficiente osmótico, $\phi$,

$\phi=-\frac{55,511 \mathrm{n} \mathrm{as}}{\Sigma_{\mathrm{i}} \vartheta_{\mathrm{i}} \mathrm{m}_{\mathrm{i}}}$

A principal vantagem reside no facto duma pequena diferença da actividade do solvente - a que corresponde, porém, uma diferença muito superior para o soluto - ser significativamente ampliada nos correspondentes valores de $\phi$, como se exemplifica na Tabela 1 .

TABELA 1

Actividade e Coeficiente Osmótico da Água

Solução aquosa $1 \mathrm{~mol} \mathrm{~kg}^{-1} \mathrm{Na} \mathrm{Cl}$, a $25^{\circ} \mathrm{C}$

\begin{tabular}{ccc}
\hline Solução & as & $\phi$ \\
\hline Real & 0,9668 & 0,936 \\
Ideal (as =xs) & 0,9652 & 0,982
\end{tabular}

A otenção de valores por via experimental para o maior número possível de soluçð̋es é altamente desejável. No entanto, tal tarefa é dificultada, ou mesmo impossibilitada, face a questð̋es processuais ou limitação temporal.

Assim, o desenvolvimento dum modelo matemático 16gico e amplamente testado é indispensável, quer como meio de confirmação quer para avaliar/prever valores de sistemas não testados.

Nesse âmbito, embora limitado na simplicidade postular [1], o modelo de Debye-Hückel equaciona o desvio da realidade gerado pelas forças iónicas, através do cálculo do trabalho, W, necessário para carregar electricamente o equivalente sistema ideal, mantendo a mesma distribuição. Com base num raciocínio formalmente electrostático, usa a lei da distribuição de Boltzmann para determinar a distribuição média da carga na vizinhança de um ião. Recorrendo a necessárias simplificaçס̃es matemáticas e aproximaçð̃es de natureza física que obrigam a assumir um carácter de extrema diluição para a solução, obtém-se

$\Delta \mu=\mu-\mu^{\mathrm{o}}=\mathrm{W}=\mathrm{L} \iint_{\mathrm{o}}^{z_{\mathrm{i}}} \boldsymbol{\Psi}(\mathrm{o}) \mathrm{dq}$,

onde $\Psi(0)$ representa o potencial produzido num ião de referência pelos restantes presentes na solução.

Atendendo a (3),

ln $\mathrm{f}=\frac{\mathrm{W}}{\mathrm{RT}}$

que por dedução matemática [1] conduz a

$\log f_{i}=-\frac{A Z_{i}^{2} I^{1 / 2}}{1+B a I^{1 / 2}}$,

onde i denota uma espécie iónica de carga $Z_{\mathrm{i}} \mathrm{e}$.

A equação (19) converte-se na usual expressão de utilização prática, com validade razoável até $\mathrm{I}=0,1$ $\log \mathrm{f}_{ \pm}=-\mathrm{Z}_{+}\left|\mathrm{Z}_{-}\right| \frac{\mathrm{A} \mathrm{I}^{1 / 2}}{1+\mathrm{Ba} \mathrm{I}^{1 / 2}}$

Para soluções fortemente diluídas, $\mathrm{Ba} \mathrm{I}^{1 / 2} \ll 1$ $\left(\mathrm{I} \leq 10^{-3}\right)$, tem-se a conhecida lei limite

$\log \mathrm{f}_{ \pm=}=-\mathrm{Z}_{+}\left|\mathrm{Z}_{-}\right| \mathrm{A} \mathrm{I}^{1 / 2}$

Deve colocar-se a questão: são as contantes A e B independentes da escala de concentraçð̃es adoptada para o cálculo da força iónica I?

A Tabela 2 mostra que não, chamando a atenção para a coerência com a relação $\mathrm{c} \approx \varrho \mathrm{m}$, válida em soluçð̃es muito diluídas. A Tabela 3 concretiza e exemplifica a inferência.

TABELA 2

Cálculo de $A$ e $B$ nas escalas práticas de concentração

\begin{tabular}{cc}
\hline Molal $(\mathrm{m})$ & Molar $(\mathrm{c})$ \\
$\mathrm{I}=\frac{1}{2} \sum_{\mathrm{i}} \mathrm{m}_{\mathrm{i}} \mathrm{Z}_{\mathrm{i}}{ }^{2}$ & $\mathrm{I}=\frac{1}{2} \sum_{\mathrm{i}} \mathrm{m}_{\mathrm{i}} \mathrm{Z}_{\mathrm{i}}{ }^{2}$ \\
$\mathrm{~A}=\frac{1,825 \times 10^{6}}{(\epsilon \mathrm{T})^{3 / 2}} \sqrt{\mathrm{eS}}$ & $\mathrm{A}=\frac{1,825 \times 10^{6}}{(\epsilon \mathrm{T})^{3 / 2}}$ \\
$\mathrm{~B}=\frac{50,29}{(\epsilon \mathrm{T})^{1 / 2}} \sqrt{\mathrm{eS}}$ & $\mathrm{B}=\frac{50,29}{(\epsilon \mathrm{T})^{1 / 2}}$ \\
\hline
\end{tabular}

TABELA 3

Constantes da equação de Debye-Hückel a várias temperaturas [2]

\begin{tabular}{|c|c|c|c|c|}
\hline \multirow[b]{2}{*}{$t \% \mathrm{C}$} & \multicolumn{2}{|c|}{ A } & \multicolumn{2}{|c|}{ B } \\
\hline & conc/mol dm${ }^{-3}$ & conc/mol kg ${ }^{-1}$ & conc/mol dm ${ }^{-3}$ & conc/mol kg \\
\hline 15 & 0.5002 & 0.5000 & 0.3267 & 0.3266 \\
\hline 25 & 0.5092 & 0.5085 & 0.3286 & 0.3281 \\
\hline 35 & 0.5190 & 0.5175 & 0.3307 & 0.3297 \\
\hline 45 & 0.5296 & 0.5270 & 0.3330 & 0.3314 \\
\hline 55 & 0.5410 & 0.5371 & 0.3353 & 0.3329 \\
\hline 65 & 0.5534 & 0.5480 & 0.3379 & 0.3346 \\
\hline 75 & 0.5668 & 0.5596 & 0.3406 & 0.3363 \\
\hline 85 & 0.5814 & 0.5722 & 0.3434 & 0.3380 \\
\hline 95 & 0.5972 & 0.5857 & 0.3466 & 0.3399 \\
\hline
\end{tabular}

Mas, mais importante é realçar a incorrecção, vulgarmente verificada, de considerar o coeficiente de actividade calculado por (20) ou (21) como o correspondente à escala utilizada na determinação de $\mathrm{I}$, isto é

$\mathrm{m} \bumpeq \gamma_{ \pm}$

$\mathrm{c} \hat{=} \mathrm{y}_{ \pm}$

o que é obviamente incorrecto pois, independentemente da escala usada para a força iónica (e respectivas constantes A e B), o coeficiente de actividade obtido será sempre $o$ correspondente à fraç̧ão molar $f \pm$.

Com efeito, a teoria determina a variação do potencial químico referente às interacçð̋es ião-ião relativamente à solução ideal.

Exemplificando para a escala molal

$\mu=\mu$ 旦 $+\vartheta \mathrm{RT} \ln \vartheta_{+} \gamma_{ \pm} \frac{\mathrm{m}}{\mathrm{m}_{\mathrm{o}}}$

onde $\mathrm{m}_{\mathrm{o}}=1 \mathrm{~mol} \mathrm{~kg}^{-1}$.

Uma vez que $\gamma_{ \pm}=f_{ \pm} x_{S}[3]$

$\mu=\mu$ 昷 $+\vartheta \mathrm{RT} \ln \vartheta_{ \pm} \mathrm{f}_{ \pm} \mathrm{xs}_{\mathrm{s}} \frac{\mathrm{m}}{\mathrm{m}_{\mathrm{o}}}$ 
sendo

$\mu^{\circ}=\mu_{\mathrm{m}}^{\ominus}+\vartheta \mathrm{RT} \ln \vartheta_{ \pm} \times \mathrm{S}_{\frac{\mathrm{m}}{\mathrm{m}_{\mathrm{o}}}}$

$\mu-\mu^{\circ}=\vartheta \mathrm{RT} \ln \mathrm{f}_{ \pm}$

A “confusão" [4,5] advém de, nas escalas práticas, se considerar como potencial químico da solução ideal

$\mu^{\circ}=\mu_{\mathrm{m}}^{\ominus}+\vartheta \mathrm{RT} \ln \vartheta_{ \pm} \mathrm{m}$

ou

$\mu^{\circ}=\mu_{\mathrm{c}}^{\ominus} \vartheta \mathrm{RT} \ln \vartheta_{ \pm} \mathrm{c}$

em vez da expressão correcta, relação (24) ou equivalente para o caso da escala molar.

Embora, na aplicação a soluções muito diluídas

- o limite de validade da teoria seja $10^{-2} \mathrm{~mol} \mathrm{~kg}^{-1}$

- e em solvente aquoso, se obtenha

$\mathrm{f}_{ \pm} \approx \gamma_{ \pm} \approx \mathrm{y}_{ \pm}$

como se exemplifica para um electrólito simétrico com $\mathrm{m}=10^{-2} \mathrm{~mol} \mathrm{~kg}^{-1}$

$\gamma_{ \pm}=\mathrm{f}_{ \pm} \mathrm{x}_{\mathrm{S}}=\mathrm{f}_{ \pm} \frac{1}{\vartheta \mathrm{m} \mathrm{M}_{\mathrm{S}}+\overline{1}} 0,9996 \mathrm{f}_{ \pm}$

importa explicitar perfeitamente o rigor do conceito, evitando eventuais erros em situações diversas dos casos mais vulgares, nomeadamente:

Determinação correcta do coeficiente de actividade quando se utilizam leis estendidas

$$
\mathrm{f}_{ \pm}=-\mathrm{Z}_{+} \mid \mathrm{Z} \downarrow \frac{\mathrm{A} \mathrm{I} \mathrm{I}^{1 / 2}}{1+\mathrm{Ba} \mathrm{I}^{1 / 2}}+\mathrm{CI}
$$

aplicáveis a soluções mais concentradas

Ex.: $\mathrm{NaCl}, 1 \mathrm{~m}, \gamma_{ \pm}=0,9653 \mathrm{f}_{ \pm}$

Soluções em solventes orgânicos de elevada massa molecular

Ex.: $\mathrm{CH}_{3} \mathrm{CONHCH}_{3}, 0,03 \mathrm{~m}, \gamma_{ \pm}=0,9959 \mathrm{f}_{ \pm}$

Uso de modelos mais sofisticados aplicáveis a casos particulares e cobrindo concentrações elevadas

Ex.: Electrólito simétrico, meio aquoso, $\mathrm{m}=2$, $\gamma_{ \pm}=0,9328 \mathrm{f}_{ \pm}$

Referências

[1] J.O'M. Bockris e A.K.N. Reddy, "Modern Electrochemistry", Plenum, 3. a ed. (1972)

[2] G. Kortum, "Treatise on Electrochemistry", Elsevier, Amesterdam, 2." ed. (1965)

[3] Luisa M.D.A. Abrantes e M. Filomena G.F.C. Camðes, «Soluçōes Iónicas: Sobre os Conceitos de Estado Padrão e Potencial Padrão de Eléctrodon, Submetido à Rev. Port. Quím.

[4] J.P. Morel, J. Chem. Educ., 57, 246 (1980)

[5] G. Jaucso e D.V. Feuby, J. Chem. Educ., 60, 382 (1983)

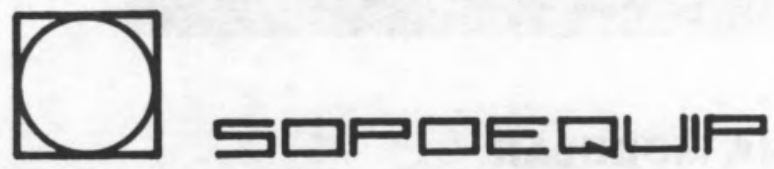

PRODUTOS E EQUIPAMENTOS PARA A INDÚSTRIA E LABORATORIOS LDA

\section{DINAMISMO - QUALIDADE \\ SERVIÇO}

\section{ESCOLHA - EFICIÊNCIA}

PEÇA-NOS A LISTA DAS NOSSAS REPRESENTADAS

ALGUMA LHE INTERESSARÁ!

Estamos à distância do seu telefone...

QUINTA DA PIEDADE, LOTE $12 \cdot 1 .^{\circ}$ 


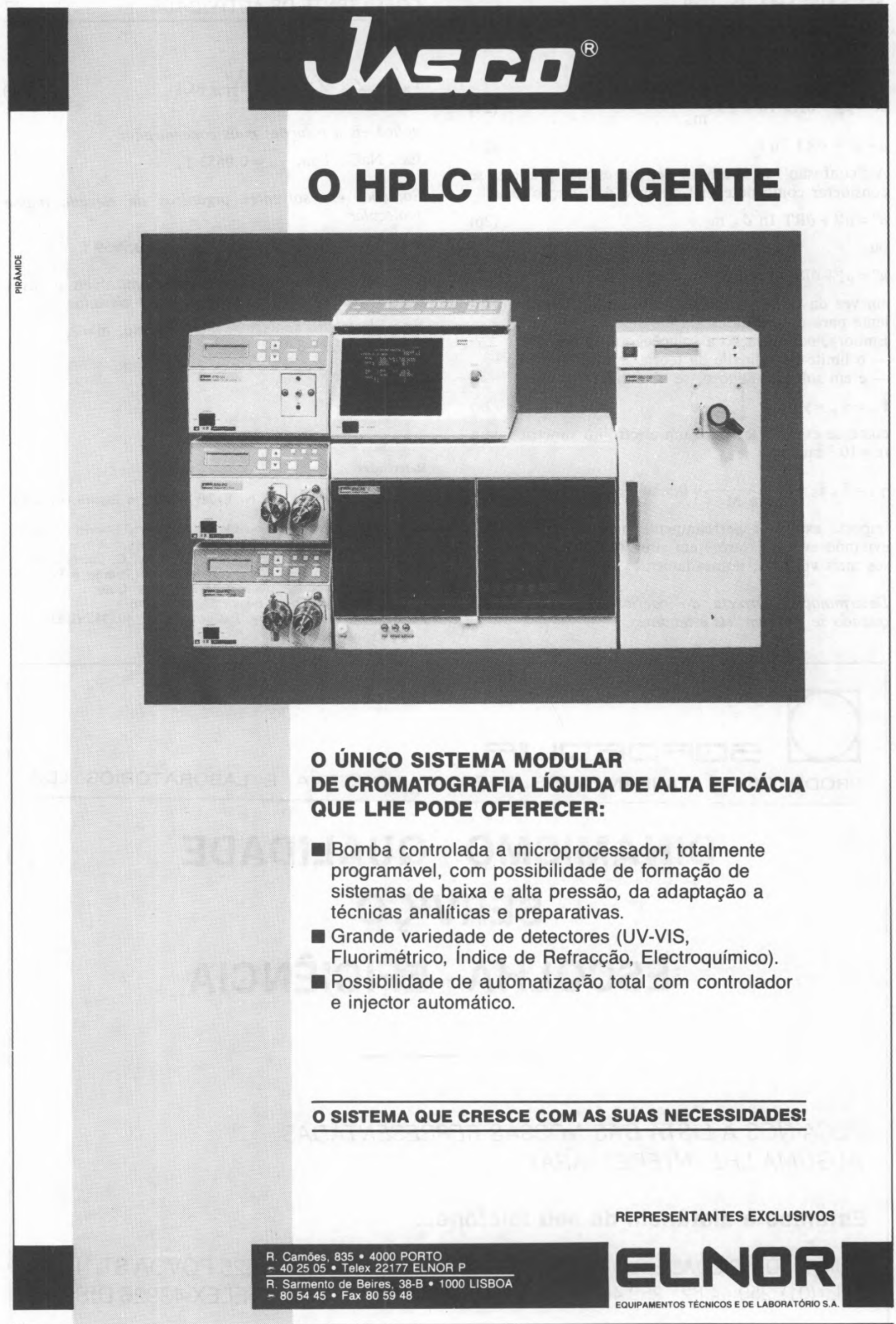

